\title{
Comparison of the High Pressure Structures of Spinel Single Crystals with Composition $\mathrm{Mg}_{0.4} \mathrm{Al}_{2.4} \mathrm{O}_{4}$ and $\mathrm{ZnCr}_{2} \mathrm{~S}_{4}$
}

\author{
J. Wittlinger, S. Werner and H. Schulz
}

Institut für Kristallographie und Angewandte Mineralogie der Universität München,

Theresienstr. 41, D-80333 München, Germany

\begin{abstract}
The present study deals with single crystal structure investigations of $\mathrm{Mg}_{0.4} \mathrm{Al}_{2.4} \mathrm{O}_{4}$ and $\mathrm{ZnCr}_{2} \mathrm{~S}_{4}$ by means of $\mathrm{X}$ ray scattering. The compressibility of the oxide substance is very small, whereas the structural changes are surprisingly high. In contrast, the compressibility of the thiospinel is 3 times higher, but the structural changes are very small. The transition to a non-detectable high pressure phase of $\mathrm{Mg}_{0.4} \mathrm{Al}_{2.4} \mathrm{O}_{4}$ turns out to be reversible considering the existence of a spinel single crystal, but not reversible concerning the values of the lattice constant and the positional parameter of the oxygen anion. This can be explained by pressure induced disordering of the cations, which is preserved on abrupt pressure decrease.
\end{abstract}

[single crystal, spinel, phase transition, high pressure]

\section{Introduction}

The representatives of the spinel group owe a wide range of chemical composition and a high symmetry (space group Fd $\overline{3} \mathrm{~m}$ ) [1]. The common chemical formula is $\mathrm{AB}_{2} \mathrm{X}_{4}$ where $\mathrm{A}$ and $\mathrm{B}$ denote the tetrahedrally and octahedrally coordinated cations, respectively, and $\mathrm{X}$ denotes the anion. These ternary compounds reveal a large variety of physical properties, according to the individual composition. Therefore they seem to be ideal to investigate the interaction of the influence of structure and chemical composition to the response on external fields such as heat, pressure, magnetism etc. In addition, investigations of spinels under pressure have a geophysical implication, following the hypothesis of the layering of the earth's mantle which is in a main part explained by the subsequent phase transitions of olivine-like compounds passing the intermediate stage of spinels to result by further increase of pressure and temperature in a perovskite-like structure. Both of these phase transitions, which are supposed to be of first order, are accompanied by an increase in density of several percent. This difference in weight can explain the layered structure of the earth's mantle, which is supposed to exist according to seismological observations of the earths interior. As the mantle material should convect, the phase transitions in question are expected to be reversible. The spinel-perovskite phase transition today is mostly identified with a step in seismic velocities at a depth of about $670 \mathrm{~km}$ in the earth's interior [2]. Powder diffraction experiments under high pressure and high temperature on $(\mathrm{MgFe})_{2} \mathrm{SiO}_{4}$ spinel confirm the existence of the assumed phase transition to a perovskite-like phase under mantle conditions [3].

So far experiments under pressure mainly dealt with oxide spinels, but also thiospinels have been investigated. Single crystal diffraction experiments on oxide spinels up to now did not reach the relevant pressure regions to get information about phase transitions, only changes in lattice constants and in the positional parameters could be observed. A tendency seems to exist, that all positional parameters under pressure approach the value where the cation packing would be an ideal $f c c$-structure. $\mathrm{CuIr}_{2} \mathrm{~S}_{4}$, however, shows a transition to a tetragonal phase at $2.5 \mathrm{GPa}$ [4]. The substances under consideration in this work are the prototype of the spinel group, $\mathrm{MgAl}_{2} \mathrm{O}_{4}$, simply called "spinel" and $\mathrm{ZnCr}_{2} \mathrm{~S}_{4}$, named "kalininite". Previous spectroscopic experiments on $\mathrm{MgAl}_{2} \mathrm{O}_{4}$ revealed a phase transition at $11 \mathrm{GPa}$ and a second one at $19 \mathrm{GPa}$, which leads to an amorphous phase [19]. The phase transitions turned out to be reversible, concerning the observed spectra.

\section{Considerations about the spinel structure}

Spinels $\mathrm{AB}_{2} \mathrm{X}_{4}$ consist of an anion framework, where the tetrahedrally coordinated positions are occupied by the cations with $1 / 8$ of all interstitial places and the octahedrally coordinated places are half occupied. Oxygen and sulphur are the most common representatives of the anion framework. The formal charge of the cations is in the case of $\mathrm{X}^{2-}$ either $\mathrm{A}^{2+}$ and $\mathrm{B}^{3+}$ or $\mathrm{A}^{4+}$ and $\mathrm{B}^{2+}$. In both cases, the sum of the formal charges results to zero. The anion framework is a nearly ideal $f c c$-structure. The space group allows only one free positional parameter, called " $u$ ", which describes the deviation of the anions from the ideal $f c c$-configuration. Here, throughout the paper, we chose the origin of the coordinate system at the center 
of inversion, and so, the parameter $u$ of the $f c c$-structure is 0.25 . Usual spinels have values of $u$ between 0.24 and 0.27 , depending on the combination of the cations and anions [1].

In real spinel structures, the distribution of the cations often is not as ideal as described here, but the stoichiometry is different, and the cations are disordered on their sites. The disordering of the cations can be described by means of a parameter $\lambda$, leading to the more general formula $B_{\lambda} A_{1-\lambda}\left(A_{\lambda} B_{2-\lambda}\right) X_{4}$ where the occupation of the octahedral position is in brackets. With $\lambda=0$ the ideal distribution $\mathrm{A}\left(\mathrm{B}_{2}\right) \mathrm{X}_{4}$ is obtained, with $\lambda=1$, complete inversion can be described with the formula $\mathrm{B}(\mathrm{AB}) \mathrm{X}_{4}$. Ideal stochastic distribution corresponds to the value $\lambda=2 / 3$ [5].

\section{Experimental}

The $\mathrm{MgAl}_{2} \mathrm{O}_{4}$ samples were small chips, taken from a piece of spinel, produced with the verneuil method ${ }^{1} . \mathrm{ZnCr}_{2} \mathrm{~S}_{4}$ was synthesized by firing of $\mathrm{ZnS}$ and $\mathrm{CrCl}_{3}$ for 12 days at a temperature of $T=1100 \mathrm{~K}[6]$. Before the beginning of the high pressure experiments, great care was taken to characterize the probes. For every individual sample rocking curves were investigated to get information about the mosaicity of the sample, and in addition the existence of twin domains following the spinel law were excluded. Especially the thiospinels were very brittle and showed heavy twinning

The high pressure experiments were performed with singlecrystal diamond anvil cells, which allow a maximum pressure of more than $30 \mathrm{GPa}$ and show an opening angle of $100^{\circ}$ on both sides, transparent for $\mathrm{x}$-rays [7]. Thus, for cubic structures in most positions of the crystal in the cell, nearly complete datasets can be collected up to $\sin \theta / \lambda=0.8$ or higher. The culet diameter was between 0.4 and $0.6 \mathrm{~mm}$ and the pressure chamber had a height of $90 \mu \mathrm{m}$ and a diameter between 150 and $300 \mu \mathrm{m}$, depending on the culet used in the individual high pressure run. Typical sample sizes were about $30 \mu \mathrm{m}$ in height and $40 \mu \mathrm{m}$ in diameter. The pressure transmitting medium was either argon, which was loaded cryogenically [8], or an alcohol mixture. Argon was used in the experiments which were dedicated to reach pressures which exceed the limit of hydrostaticity of the alcohol mixture. Pressure was measured in situ by the ruby fluorescence method, using a small single crystal of ruby which was introduced in the pressure chamber. All experiments on the oxide spinel were done on a CAD4-four circle diffractometer with kappa geometry equipped with a sealed tube, using Mo-K $\alpha$ radiation. Only one set of data ( $p=1.9 \mathrm{GPa}$ ) was obtained at Hasylab (Hamburg, Germany), at beamline D3 ( $\lambda=0.6 \AA$ ). Lattice constants of $\mathrm{ZnCr}_{2} \mathrm{~S}_{4}$ were obtained, both on the CAD4 Diffractometer and at Hasylab whereas intensity datasets were all obtained at Hasylab, except the one at ambient pressure. To center the cell on the diffractometer, the profile of the primary beam was used, what can provide an accuracy comparable to the sphere of confusion

\footnotetext{
${ }^{1}$ We greatfully acknowledge Fa. Djevahirdjian, Monthey (Switzerland) for the spinel-probe.
}

of the diffractometer. In general, the lattice constants were obtained by centering 12 or more reflections at $\theta>10^{\circ}$, mostly at 4 positions $( \pm \theta, \pm \chi)$. Intensity data were collected with $\omega$ scans and every independent reflection set was measured at least 3 times.

Data were reduced with the program REDA, dedicated to handle high pressure data. Herein, empiric absorption correction of the reflections, normalizing of the intensities to the primary beam monitoring system (not available for CAD4data), and Lorentz polarisation correction can be performed. Additionally this program allows to integrate the reflections automatically, and to see the results of the integration in a graphic display, showing all (maximum number 9) symmetrically equivalent reflections simultaneously. It is also possible, to set the integration limits manually in an interactive procedure. This is only necessary for weak reflections $(\mathrm{I} / \sigma(\mathrm{I})<2)$ where the automatic integration procedure obviously fails. Structure refinements were all done with SHELXL93.

\section{Results}

\section{$\mathrm{MgAl}_{2} \mathrm{O}_{4}$}

\section{Sample characterisation}

The characterisation of the substance at ambient conditions shows a lattice constant of $\mathrm{a}=7.978(2) \AA$ and a positional parameter of $u=0.2580(1)$. Using these values, the chemical formula of the real structure turned out to be $\mathrm{Mg}_{0.4} \mathrm{Al}_{2.4} \mathrm{O}_{4}[9]$. According to previous works, this leads to a typical disordering of the Al-cations on the two different sites of the structure, and an ordering of the $\mathrm{Mg}$-ions on the tetrahedral site [9]. These values are also in good agreement with the site occupations, calculated by use of the electron densities of the different positions, assuming a globally neutral structure and an ordering of the $\mathrm{Mg}$-ions on the tetrahedral site. The symmetrical implications of the structure lead to the result, that the tetrahedron always shows an ideal geometry, whereas the bond angles of the octahedron are dependent on the positional parameter $u$. At the value $u=0.25$, the octahedron would be perfectly symmetric with bond angles $\mathrm{M}-\mathrm{O}-\mathrm{M}=90^{\circ}$ (M="metal"). Here, the octahedron is squeezed along its threefold axis.

\section{Lattice constants and equation of state}

As already revealed in previous experiments, the bulk modulus of spinel is very large and thus, the relative volume change under pressure is small [10]. This leads to considerable relative errors in the determination of the lattice constants used for the calculation of the equation of state. As no systematic difference in the individual high pressure runs, nor a dependence of the results on the use of different pressure media (alcohol or argon) could be observed, all obtained data were used to determine one averaged equation of state. The pressure 
range is limited due to a phase transition that occurs at a pressure between 10.5 and $11 \mathrm{GPa}$. Above the phase transition, no lattice constants could be obtained, because the signals vanished and the reflections broadened abruptly. The rectangle in Fig. 1 denotes the lattice constant of a sample which had been recovered from a high pressure run up to a maximum pressure of $15 \mathrm{GPa}$, which exceeded the phase boundary.

The equation of state agrees within the errors with previous experiments, which were done up to ca. $4 \mathrm{GPa}$ on a stoichiometric, natural sample [9]. Thus, no dependence of the compressibility of the substance on the disordering of the cations can be observed. In comparison to other oxide spinels

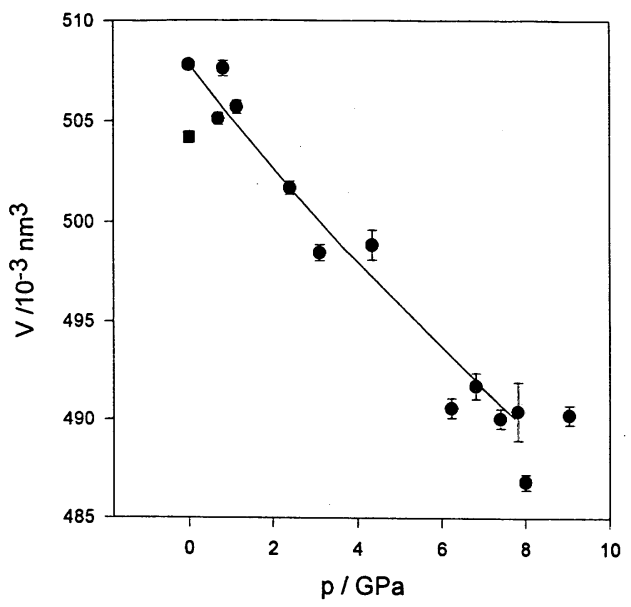

Fig. 1 Pressure induced volume change of spinel. The fitted line represents a Birch-Murnaghan equation of state. $\square$ denotes a specimen which supported a maximum pressure of $15 \mathrm{GPa}$ for several hours.

investigated under pressure so far, it can be stated, that the bulk moduli only show small differences, hardly beyond the reported errors (s. Tab. 1). The rectangle, originating from the sample recovered from the high pressure region, shows that the phase transition is in some aspects irreversible (see below for details).

\section{Pressure dependence of the structure}

Despite the small change in volume, the structural changes of spinel under pressure are considerable and show some interesting features. The positional parameter $u$ approaches the value of 0.25 , but not in a linear pressure dependence, as could be concluded of previous studies. Here, the compression seems to be nearly isotropic up to $5 \mathrm{GPa}$, and with further pressure increase, the slope changes rapidly. Between 10.5 and $11 \mathrm{GPa}$, the value of $u=0.255$ is reached and the structure undergoes a phase transition to a phase which shows no single crystal reflections. The halfwidth of the reflections increases abruptly

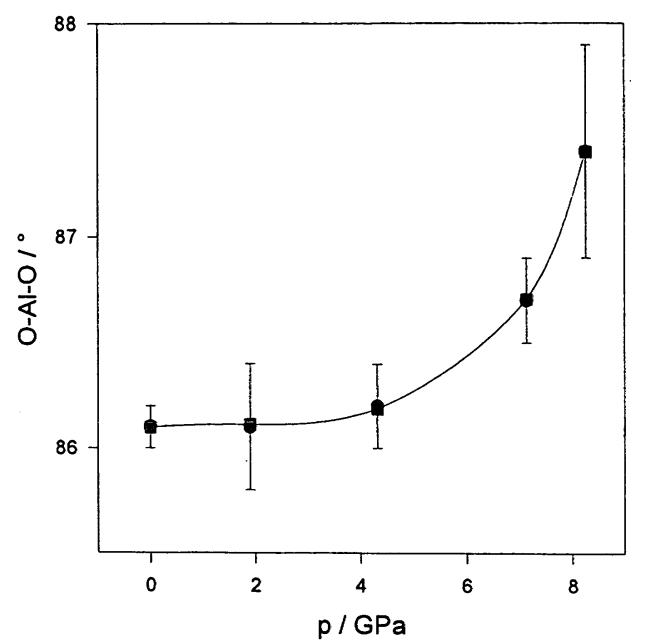

Fig. 2 Variation of the octahedron bond angle of spinel under pressure. In an ideal fcc-framework, the value would be $90^{\circ}$. The curve is a fitted exponential function.

at the transition point and results in a signal which is smeared out in $\omega$ and thus becomes unobservable with conventional single crystal methods. On complete pressure release, reflections can be detected again, but with smaller intensities as the initial ones $\left(I_{0} / I=5\right)$ and a halfwidth which is increased by a factor 1.5 on a CAD4 diffractometer. Thus, the single crystal, at least partially, seems to be reconstructed. It can be observed that the value of $u$ is not completely reversible, but it remains at a point which corresponds to about $5 \mathrm{GPa}$ on the initial pressure scale.

The volumes of the coordination polyhedra depend both on the lattice constant and on the positional parameter. The results are shown in Fig. 2 and Fig. 3. Obviously, the compressibility of the octahedron is the same over the whole pressure range in consideration, and also nearly the same as for the tetrahedron up to $5 \mathrm{GPa}$. At higher pressures, the tetrahedron shows a higher compressibility, leading to a considerable larger volume decrease compared to the octahedron. At the same time, the bond angle of the octahedron shows a rapid change above $5 \mathrm{GPa}$ (s. Fig. 2).

\section{Phase transition at $11 \mathrm{GPa}$}

In our experiments, a phase transition occured at a pressure of about $10.7 \mathrm{GPa}$. The reflections broaden suddenly, and on further pressure increase the probe appears amorphised, what means here, that no single crystal reflections could be detected anymore by means of photographic and scintillation counter techniques. At the onset of the phase transition, the overall 
volume of the structure corresponds to $97 \%$ of the initial one, whereas the volume reduction of the two coordination polyhedra differs significantly (s. Fig. 3). The positional parameter u shows a value near 0.255 , which corresponds to the break-even of the configurational energy for the cation ordering

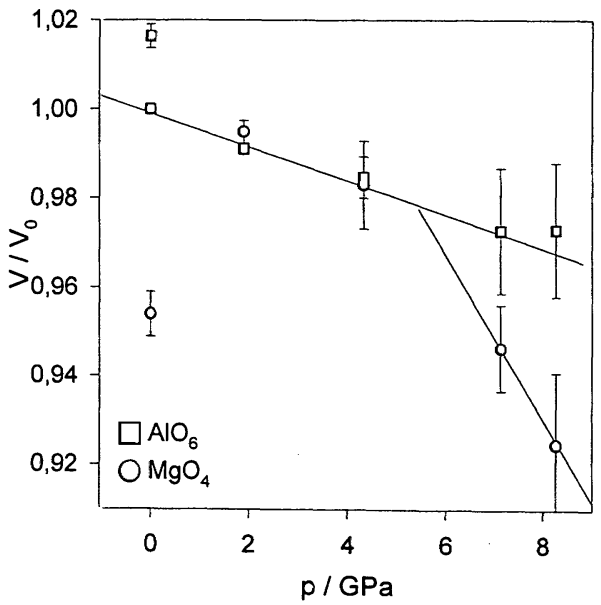

Fig. 3 Pressure dependence of the volumes of the coordination polyhedra in spinel. The values at ambient pressure different from 1.0 originate from a recovered sample that beared a maximum pressure of $15 \mathrm{GPa}$.

and disordering on the different sites of the structure [11]. On complete pressure release, an investigation of a sample which beared $15 \mathrm{GPa}$ maximum pressure for several hours leads to a lattice constant which is slightly smaller than the initial one and also the value of $u$ is not completely reversible but remains at a value which corresponds to about $5 \mathrm{GPa}$ in the initial highpressure experiment. The positional parameter $u$ is dependent on the cation ordering or disordering, following the equation (i), where $\mathrm{R}$ represents the relation between the distances of the octahedral $(\mathrm{O}-\mathrm{X})$ and tetrahedral $(\mathrm{T}-\mathrm{X})$ bond lengths (equ. ii). These distances are on the other hand only dependent on the parameter of inversion, $\lambda$, and the bond distances of the first cation "A" and the second cation "B" in their octahedral and tetrahedral coordination with oxygen, here denoted with the suffixes " $\mathrm{O}$ " and " $\mathrm{T}$ ".

$$
\begin{array}{ll}
u(R)=0,25 R^{2}-\frac{2}{3}+\frac{\sqrt{\frac{11 R^{2}}{48}-\frac{1}{18}}}{2 R^{2}-2} & \text { equ. i } \\
R=\frac{(O-X)}{(T-X)} & \text { equ. ii } \\
R=\frac{0,5 \lambda d\left(A_{O}\right)+(1-0,5 \lambda) d\left(B_{O}\right)}{\lambda d\left(A_{T}\right)+(1-\lambda) d\left(B_{T}\right)} & \text { equ. iii }
\end{array}
$$

So, the change in $u$ can be explained by a change in the cation distribution. Before the high pressure experiment, the $\mathrm{Mg}^{2+}$ cations are ordered on the tetrahedral sites and this ordering corresponds to $u=0.2580(1)$. After the high pressure experiment, the resulting value of $u=0.2569$ (2) corresponds to a partial disordering on the different polyhedron sites, namely $\lambda=0.17(2)$. The use of this formalism suggests, that after the phase transition to the high pressure phase, the cations change their positions in the oxygen framework and, at least partially, keep this position after pressure release. The slight decrease of the lattice constant also indicates that the crystal is in a state, different from the initial one. Reports from high-temprerature structure investigations [12] and theoretical considerations [13] on spinels show a comparable effect of disordering under the influence of heat. The theoretical work revealed a decrease in the lattice constant on disordering, what is in agreement with this experiment whereas other experimental results show an increase [14]. So, no final conclusion can be made about the change of the lattice constants, the results remain contradictory. It only can be stated, that there is a dependence of the lattice constant on the cation distribution.

$\mathrm{ZnCr}_{2} \mathrm{~S}_{+}$

\section{Sample characterisation}

$\mathrm{X}$-ray investigations of different crystals at ambient pressure resulted in a unit cell volume which is in agreement with that reported from powder diffraction experiments [15]. No differences between the individual samples were observed, which suggests that the specimens are of homogeneous composition. Again different high pressure runs show no systematic differences between alcohol or argon as pressure medium or between conventional tube-measurements and synchrotron-measurements. Therefore all values are used to determine one equation of state of the $\mathrm{ZnCr}_{2} \mathrm{~S}_{4}$-spinel (Fig. 4) A least squares fit of a Birch-Murnaghan equation of state leads to the values:

$$
\begin{aligned}
& \mathrm{K}=76(9) \mathrm{GPa} \\
& \mathrm{K}^{\prime}=12(4)
\end{aligned}
$$

The value of $\mathrm{K}$ is in the range of silicate framework structures and is about a third of that of oxide spinels. This is 
astonishing small for such a compact structure as spinel, indicating a relatively high compressibility of the sulphur ions themselves.

$\mathrm{K}^{\prime}$, in contrast, is relatively high, usual values are around 4 . Fitting the equation of state with the constraint $K^{\prime}=4$, leads to a value of $\mathrm{K}=97(10) \mathrm{GPa}$.

The two triangles and the square in Fig. 4 denote unit cell volumes of samples that have been recovered from high pressure runs with a maximum pressure where the reflections of the samples had already vanished. The measured intensities of the corresponding reflections were very weak (approx. $10 \%$ of the initial ones). The final volume is in general considerably smaller than the initial one, so either a phase transition in the sample took place, or a hysteresis effect lead to a plastic

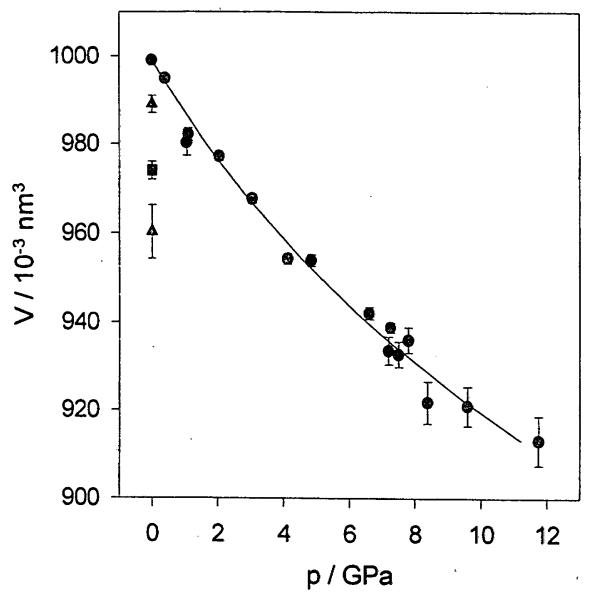

Fig. 4 Volume change of $\mathrm{ZnCr}_{2} \mathrm{~S}_{4}$ under pressure. The fitted line represents a Birch-Murnaghan equation of state. The two denote values of recovered samples with a maximum pressure of 8 (lower) and $12 \mathrm{GPa}$ (upper), respectively. denotes a value of a specimen which beared a maximum pressure of $21 \mathrm{GPa}$.

deformation of the single crystal, or a decomposition of different chemical constituents began to take place, leading to a resulting sample with an inhomogeneous chemical composition (E. Hinze, personal communication). The obtained values show that the effect is strongly dependent on the individual sample. The maximum pressure and the resulting volume seem to be independent.

\section{Structure refinements}

Structure determination of the substance at ambient pressure resulted in a value for the sulphur 1positional parameter of $\mathrm{u}=0.25958(3)$. According to the symmetries of spinel type structures, the $\mathrm{CrS}_{6}$ octahedron is stretched along the (111)-

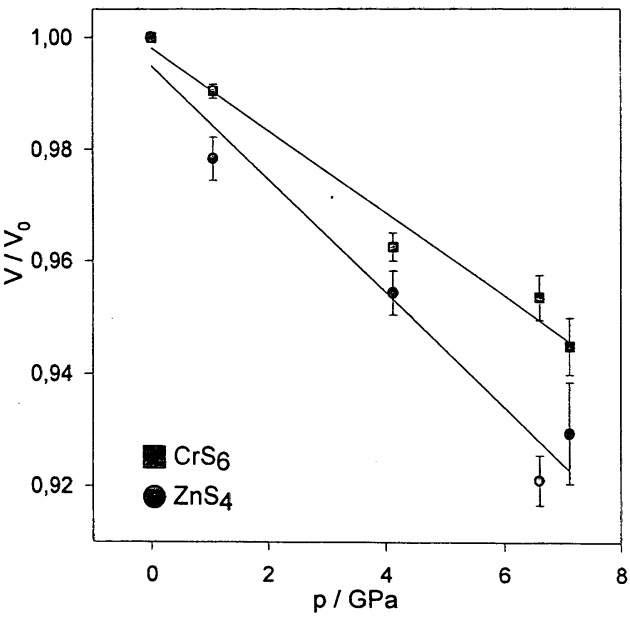

Fig. 5 Pressure dependence of the volumes of the coordination polyhedra of $\mathrm{ZnCr}_{2} \mathrm{~S}_{4}$. The lines represent linear fits of the compressibility.

axis. Site occupation factors showed values that are in accordance with an ideal configuration of the cations in the samples. The structure refinements with high pressure data were performed with occupation factors fixed at ideal values. Structure determination became more and more difficult with increasing pressure because of reflection broadening. Therefore, the errors and residuals of the structure determinations increase with pressure. Nevertheless, the results show clearly that the positional parameter " $u$ " of sulphur approaches his ideal position [16]. The tetrahedron is compressed isotropically and the octahedron approaches ideal geometry and, at the same time, the tetrahedron appears to be more compressible than the octahedron (Fig. 5). The value of the ideal $f c c$-configuration of the sulphur-anions $(u=0.25)$ would be reached at a pressure of about $100 \mathrm{GPa}$, estimated by linear extrapolation of the present data. The difference between the three values of the bulk modulus for the entire structure and the two coordination polyhedra is rather small and hardly beyond the standard deviations, but nevertheless it can be seen that there is a trend towards an ideal configuration of the anions under pressure. However, the main effect of pressure on the structure is an isotropic volume reduction.

\section{Discussion}

A comparison of the results with other structure determinations of spinel type compounds under pressure suggests, that all representatives of the spinel group have the tendency to approach the value of $u=0.25$, independently of 
the cation combinations, the nature of the anions or the starting value of $u$, which can be lower or higher than 0.25 . Magnetite, which shows a value of $u=0.25$ at ambient pressure compresses without structural changes [10]. Following this, the ideal $f c c$-configuration of the anions seems to be the favoured configuration to be reached under the influence of pressure. The response of the structure is the tendency to reach a configuration with the highest possible density. This fact is also known for other structures under pressure [17]. The non- linear dependency of the parameter $u$ of $\mathrm{Mg}_{0.4} \mathrm{Al}_{2.4} \mathrm{O}_{4}$ on the applied pressure is a feature rarely observed for spinels up to now. Only experiments on $\mathrm{Ni}_{2} \mathrm{SiO}_{4}$ indicate a comparable behaviour, but unfortunately the maximum pressure reached in that experiment was too low to get the required information unequivocally. Other structure investigations did not reach the relevant pressure regions to observe an indication for a non-linear behaviour of the positional parameter.

A phase transition of spinel under room temperature and high pressure was predicted by quantum mechanical calculations for the same pressure value as deduced here [18]. However, the expected division into $\mathrm{Al}_{2} \mathrm{O}_{3}$ and $\mathrm{MgO}$ is incompatible with the here found restructuration of the single crystal on pressure release. To undergo this transition, the single crystal should locally divide up in two different compounds, of which both form a new nucleus of a crystal. This would destroy the single crystal completely because of the change in stoichiometry. It is hard to imagine, that on pressure release

\begin{tabular}{lllll} 
specimen & $\mathrm{Mg}_{0.4} \mathrm{Al}_{2.4} \mathrm{O}_{4} \mathrm{Fe}_{3} \mathrm{O}_{4}[10]$ & $\mathrm{Ni}_{2} \mathrm{SiO}_{4}[10] \mathrm{ZnCr}_{2} \mathrm{~S}_{4}$ \\
\hline $\mathrm{K} / \mathrm{GPa}$ & $212(5)$ & $183(5)$ & 220 & $94(9)$ \\
$\mathrm{K}^{\prime} / \mathrm{GPa}$ & $6,3(1,5)$ & 5.6 & 4 & 4 \\
$\mathrm{~K}_{\text {tet }} / \mathrm{GPa}$ & $110(25)$ & $190(20)$ & $>250$ & $107(17)$ \\
$\mathrm{K}_{\text {okt }} / \mathrm{GPa}$ & $330(40)$ & $190(20)$ & $170(10)$ & $127(17)$ \\
\hline
\end{tabular}

Tab. 1 Comparison of bulk moduli of different spinel structures. All oxide spinels have comparable overall bulk moduli, whereas the thiospinel is much more compressible.

there would be a restructurisation of the single crystal after the constitution of such a high pressure phase. The high pressure phase must, in contrast, preserve in some way the original ordering of the atoms, to have the possibility to end in a new single crystal on pressure release. Independently of the calculations, spectroscopical experiments showed a phase transition at $11 \mathrm{GPa}$ and an amorphisation at $19 \mathrm{GPa}$, but that experiment was done with alcohol as pressure medium, and the point of the first phase transition was suspected to be the solidus of the pressure medium [19]. Now, the existence of the transition can be confirmed with our experiments. On the other hand, as the spectra did not vanish, but only changed their pressure dependence, this supports the fact, deduced from the reversibility of the single crystal, that the amorphisation is only some destruction of a long-range order. The x-ray experiment results in an amorphisation, but the remaining of a short-range order at a phase transition can further be observed in the spectroscopic experiments. At pressure release, this short range order can help to reconstruct the single crystal. On the other hand, experiments made on spinels, heated to more than $1000 \mathrm{~K}$ at $18 \mathrm{GPa}$, confirm the existence of the decomposition to $\mathrm{Al}_{2} \mathrm{O}_{3}$ and $\mathrm{MgO}$ [20]. Following this, the high pressure phase found here would be a metastable one, and the tranmsitiion is kinetically suppressed.

\section{References}

[1] R. J. Hill, J. R. Craig, G. V. Gibbs Phys. Chem. Min. 4 317 (1979)

[2] B. J. Wood Nature 341278 (1989)

[3] E. Ito, E. Takahashi J. Geophys. Res. 94 B 10637 (1989)

[4] T. Kagayama, G. Oomi, I. Yoshida, Y. Sakurai, T. Hagino, S. Nagata Proceedings of the joint XV AIRAPT \& EHPRG International Conference 487 (1996)

[5] V. S. Urusov Phys Chem Min. 9 1(1983)

[6] J. Wittlinger, Ph.D. Thesis, Universität München (1997)

[7] S. Werner, J. Kim-Zajonz, J. Wittlinger, H. Schulz Workshop on the Use of ultrashort Wavelengths, Hasylab, Hamburg, Germany (Proceedings) (1996)

[8] J.Wittlinger, R.Fischer, S.Werner, J..Schneider, H. Schulz, Acta Cryst. B $\mathbf{5 3} 745$ (1997)

[9] S. Lucchesi, A. Della Giusta Z. Krist. 209 714(1994)

[10] L. W. Finger, R. M. Hazen, A. M. Hofmeister Phys. Chem. Min. 13215 (1986)

[11] H. O’Neill, A. Navrotski (1983) Amer. Mineral. 68181

[12] T. Yamanaka, Y. Takeuchi Z. Krist. 16565 (1983)

[13] H. O'Neill, A. Navrotski Amer. Mineral. 68181 (1983)

[14] B. J. Wood, R. J. Kirkpatrick, B. Montez Amer. Mineral. 71999 (1986)

[15] G. Lapluye, L. Abello Memoires presentés a la societe chimique 1062 (1963)

[16] J. Wittlinger, S. Werner, H. Schulz, Phys. Chem. Min.24, 597 (1997)

[17] H. Sowa, J. Macavei, H. Schulz Z. Krist. 192119 (1990)

[18] M. Catti, G. Valerio, R. Dovesi, M. Causa Phys. Rev. B 49 14179 (1994)

[19] A. Chopelas, A. M. Hofmeister Phys. Chem. Min. 18279 (1991)

[20] L.G. Liu Geophys. Res. Lett. 29 (1975) 